\title{
Two Regions Responsible for the Actin Binding of p57, a Mammalian Coronin Family Actin-Binding Protein
}

\author{
Teruaki Oкu, ${ }^{a}$ Saotomo Iтон, ${ }^{a}$ Masamitsu Okano, ${ }^{a}$ Akiko Suzuki,${ }^{a}$ Kensuke Suzuki, ${ }^{b}$ \\ Shizuo Nakajin, ${ }^{a}$ Tsutomu Tsuji, ${ }^{*, a}$ William Michael Nauseef, ${ }^{c}$ and Satoshi Toyoshima ${ }^{d}$ \\ ${ }^{a}$ Hoshi University School of Pharmacy and Pharmaceutical Sciences; 2-4-41 Ebara, Shinagawa-ku, Tokyo 142-8501, \\ Japan: ${ }^{b}$ Pharmaceutical Frontier Research Laboratories, Japan Tobacco, Inc.; 1-13-2 Fukuura, Kanazawa-ku, Yokohama \\ 236-0004, Japan: ${ }^{c}$ The Inflammation Program and Department of Medicine, University of Iowa and Veterans Affairs \\ Medical Center; Iowa City, Iowa 52242, U.S.A.: and ${ }^{d}$ Pharmaceuticals and Medical Devices Evaluation Center, National \\ Institute of Health Science; 3-8-21 Toranomon, Minato-ku, Tokyo 105-8409, Japan. \\ Received August 22, 2002; accepted December 17, 2002
}

The actin-binding protein p57, a member of the coronin protein family, is expressed in a variety of immune cells. It has five WD repeats and a coiled-coil motif containing a leucine zipper, both of which are known to mediate protein-protein interactions. In order to identify the precise actin-binding regions in p57, and to assess the contribution of these structural motifs, we prepared various truncated p57 as fusion proteins with glutathione $S$-transferase (GST) and examined their actin-binding activity. A co-sedimentation assay demonstrated that $\mathrm{p} 57^{1-371}$ (C-terminal truncated p57) had the ability to bind F-actin, but p57 $7^{372-461}$ (a fragment containing the coiled-coil motif) did not. A segment consisting of the N-terminal 34 amino acids of p57 (p57 $7^{1-34}$ ) was found to bind to F-actin in the co-sedimentation assay. Furthermore, fluorescence microscopic observation showed that p5 $7^{1-34}$ was co-localized with F-actin in COS-1 cells after the transfection with the p5 $7^{1-34}$ construct. Deletion of ${ }^{10} \mathrm{KFRHVF}^{15}$, a sequence conserved among coronin-related proteins, from p57 $7^{1-34}$ abolished its actin-binding activity, suggesting that this sequence with basic and hydrophobic amino acids is crucial for p57 to bind to F-actin. However, the N-terminal deletion mutant $p 57^{63-461}$ retained the binding ability to $\mathrm{F}$-actin. This result suggests the presence of a second actin-binding region. Further deletion analysis revealed that p5 $7^{111-204}$, which includes the second and third WD repeats, also exhibited weak actin-binding activity in the co-sedimentation assay. Taken together, these data strongly suggest that at least two regions within Met-1 to Asp-34 and Ile-111 to Glu-204 of p57 are responsible for its binding to the actin cytoskeleton.

Key words actin-binding protein; coronin; cytoskeleton; WD repeats; leucine zipper

As the primary effector cells in the innate immune system, phagocytes such as neutrophils and macrophages contribute to host defense by engulfing and destroying invading microbes. ${ }^{1)}$ Various actin-binding proteins have been implicated in the regulation of actin-cytoskeleton interactions, essential for properties integral to phagocytic cell function, including chemotaxis, phagocytosis, and degranulation., ${ }^{2,3}$ As described previously, ${ }^{4}$ we have identified an immune cell-specific actin-binding protein p57 that has significant homology with coronin, an actin-binding protein of Dictyostelium discoideum. Coronin plays crucial roles in various cell functions, including cell locomotion, phagocytosis, and cytokinesis of Dictyostelium. ${ }^{5-7)}$ Homologous proteins to coronin have been identified in many eukaryotes from yeast to human, ${ }^{8-17)}$ and are assigned an important role in cell motility. ${ }^{18)}$ Recent reports have suggested that $\mathrm{p} 57$ participates as well in the formation of phagosome and its maturation in neutrophils and macrophages. ${ }^{19-21)}$

Most coronin protein family members, including $\mathrm{p} 57$, consist of $450-500$ amino acid residues and share common structural features, five WD (tryptophan-aspartic acid) repeats located at the center of the molecule and a coiled-coil motif containing a leucine zipper ${ }^{4)}$ at the C-terminus. ${ }^{8)}$ These motifs are known to mediate protein-protein interactions, ${ }^{22,23)}$ but their specific function in the coronin family of actin-binding proteins has not been elucidated. Since coronin has no significant homology with other actin-binding proteins, the region(s) responsible for the binding to actin remains undetermined. Recently, two groups have reported pu- tative actin binding regions in coronin family members of two distinct species. ${ }^{8,13)}$ In Crn1p, a coronin in budding yeast, the N-terminal part containing WD repeats, has been shown to have actin-binding activity. ${ }^{8}$ In Xcoronin, a coronin homologue in Xenopus oocytes, the deletion of N-terminal 63 amino acids or of C-terminal 65 amino acids reduced its actin-binding activity, although the WD repeats themselves were not directly involved in the binding to actin. ${ }^{13)}$ Thus, there is an argument concerning the region(s) responsible for actin binding of the coronin family of proteins.

To identify the region(s) responsible for actin binding of $\mathrm{p} 57$, we prepared various mutants of p57 in E. coli and assessed their ability to bind to actin by two approaches. We evaluated their abilities to co-sediment with F-actin after actin polymerization, and to co-localize with actin filaments in cells after transfection with the plasmid constructs for the mutant. The results obtained suggest that there are at least two regions responsible for actin binding of $\mathrm{p} 57$.

\section{MATERIALS AND METHODS}

Expression of Glutathione $\boldsymbol{S}$-Transferase (GST) Fusion Proteins of Full-Length, and Deletion Mutants of p57 Full-length and partial coding sequences of p57 were expressed in E. coli as GST fusion proteins using the vector pGEX-5X-1 (Pharmacia, Uppsala, Sweden). Expression plasmids pGEX-p57 (full-length), pGEX-p57 ${ }^{1-371}$ (Met-1 to Asp-371), and pGEX-p57 $7^{372-461}$ (Pro-372 to Lys-461) (Fig. 1 ), were prepared by restriction endonuclease digestion and 
adapter ligation. ${ }^{4)}$ Briefly, the construct for the expression of p57 was prepared by digestion of the full-length cDNA of p57 with PmaCI and SacI, and was ligated with both adapter I and adapter II (adapter I, upper strand 5'-GAT CCG AAT GAG CCG GCA GGT GGT CCG CTC CAG CAA GTT CCG CCA C-3' and lower strand 5'-GTG GCG GAA CTT GCT GGA GCG GAC CAC CTG CCG GCT CAT TCG-3'; adapter II, upper strand 5'-CCA GAA GCG CTT GGA CAG GCT GGA GGA GAC AGT CCA GGC CAA GTA GG-3' and lower strand 5'-TCG ACC TAC TTG GCC TGG ACT GTC TCC TCC AGC CTG TCC AAG CGC TTC TGG AGC T-3'). The construct for the expression of $\mathrm{p} 57^{1-371}$ was prepared by digestion of the full-length cDNA with PmaCI and $B g l \mathrm{I}$, and ligated with adapter I and adapter III (adapter III, upper strand 5'-CGG CTG AGG AGT GGC TGG CTG GGG GGT CGG GAT GCT GGG TAG G-3' and lower strand 5'-AGT GCC GAC TCC TCA CCG ACC CCC CAG CCC TAC GAC CCA TCC AGC T-3'). The construct for the expression of $\mathrm{p} 57^{373-461}$ was prepared by digestion of the full-length cDNA with $B g l \mathrm{I}$ and $S a c \mathrm{I}$, and ligated with adapter IV and adapter II (adapter IV, upper strand 5'-GAT CCC TGC CCT CA-3' and lower strand 5'-GGA CGG G$\left.3^{\prime}\right)$. The ligated fragments and adapters were subcloned into pGEX-5X-1, that had been digested with BamHI and SalI.

The expression plasmids of pGEX-p57 ${ }^{1-71}$ (Met-1 to Asp71) and pGEX-p5 $7^{1-34}$ (Met-1 to Thr-34) (Fig. 1), were generated from pGEX-p57 $7^{1-371}$ by introducing stop codons using a QuikChange ${ }^{\mathrm{TM}}$ Site-directed mutagenesis kit (Stratagene, La Jolla, CA, U.S.A.). The sequences of mutagenic primers are as follows (the substituted bases are underlined): K72Z, sense primer 5'-GGC AAG ACT GGA CGT GTG GAC IAG AAT GCG CCC ACG GTC TGT GG-3' and antisense primer 5'-CCA CAG ACC GTG GGC GCA TTC TAG TCC ACA CGT CCA GTC TTG CC-3'; and W35Z, sense primer 5'-CGT CTC ACA GAC CAC CT $\underline{\text { A GGA }}$ CAG TGG CTT CTG TGC-3' and anti-sense primer 5'-GCA

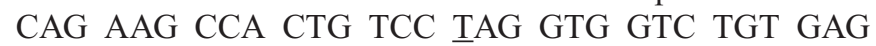
ACG-3'. Deletion of amino acid residues 10 to 15 from p $57^{1-34}$ was also performed by a QuikChange Site-directed mutagenesis kit. The primers used were: $\Delta(10-15)$, sense primer 5'-G GTG GTC CGC TCC AGC GGA CAG CCG
GCC AAG G-3' and anti-sense primer 5'-C CTT GGC CGG CTG TCC GCT GGA GCG GAC CAC C-3'.

The other deletions of p57, pGEX-p57 $7^{63-461}$ (Pro-63 to Lys-461), pGEX-p57 $63-127$ (Pro-63 to Leu-127), pGEXp57 $7^{111-204}$ (Ile-111 to Glu-204), pGEX-p57 205-296 (Pro-205 to Glu-296), and pGEX-p57 $7^{297-429}$ (Ile-297 to Ala-429) (Fig. 1 ), were obtained by polymerase chain reaction (PCR), followed by digestion and subcloning in expression vector pGEX-5X-1. The amplified DNA fragments were digested with BamHI and EcoRI, then cloned into pGEX-5X-1. The primers used are as follows (restriction enzyme sites are un-

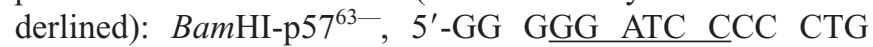
GGC AAG ACT GGA CGT GTG G-3' and EcoRI-HindIIIp5 $7^{461-}$ reverse, 5'-GGG GAA TTC AAG CTT GGG GCT CTA CTT GGC CTG G-3' for p57 $7^{63-461} ;$ BamHI-hp57 $7^{63-}$ and EcoRI-p57 ${ }^{127-}$ reverse, 5'-GGG GAA TTC CAG GGT GAC GAC GGG CTC CCG CAG G-3' for p57 ${ }^{63-127}$; BamHI-p57 ${ }^{111-}, 5^{\prime}$-GGG GGG ATC CCC ATC CCG GAT GGG GGC CTG AT-3' and EcoRI-p57 ${ }^{204}$ reverse, 5'-GGG GAA TTC CTC GAT GAT GCG CAC GCG CT-3' for p57 ${ }^{111-204} ;$ BamHI-p57 ${ }^{205-}$, 5'-GGG GGG ATC CCC CCC CGC AAA GGC ACT GTC GT-3' and EcoRI-p57 $7^{296-}$ reverse, GGG GAA TTC CTC AAA GTA CCG GAT TGA GC for p57 205-296; and BamHI-p57 ${ }^{297-}$, GGG GGG ATC CCC ATC ACT TCC GAG GCC CCT TT and EcoRI-p57 ${ }^{429-}$ reverse, GGG GAA TTC CCT CCT CCA GCC GAG ACA CG for $\mathrm{p} 57^{297-429}$.

The pGEX-p57 $7^{63-299}$ was generated from pGEX-p57 $7^{63-461}$ by introducing stop codons. The primers used were: E300Z, sense primer 5'-GGT ACT TTG AGA TCA CTT CCT AGG CCC CTT TCC TGC ACT ATC-3' and antisense primer 5'GAT AGT GCA GGA AAG GGG CCT AGG AAG TGA TCT CAA AGT ACC-3'.

Expression and Purification of GST Fusion Proteins E. coli JM109 cells, transformed with each plasmid described above, were cultured overnight in $6 \mathrm{ml}$ of LuriaBertani (LB) medium ( $10 \mathrm{~g} / 1$ of tryptone, $5 \mathrm{~g} / 1$ of yeast extract, $10 \mathrm{~g} / 1$ of $\mathrm{NaCl}, \mathrm{pH} 7.0$ ) at $37^{\circ} \mathrm{C}$, diluted to $500 \mathrm{ml}$ with fresh Terrific Broth (TB; $12 \mathrm{~g} / 1$ of tryptone, $24 \mathrm{~g} / 1$ of yeast extract, $0.8 \%$ of glycerol and $1.25 \mathrm{~mm}$ of potassium phosphate buffer, $\mathrm{pH} 6.5$ ), and grown for $48 \mathrm{~h}$ at $20^{\circ} \mathrm{C}$. Fusion proteins

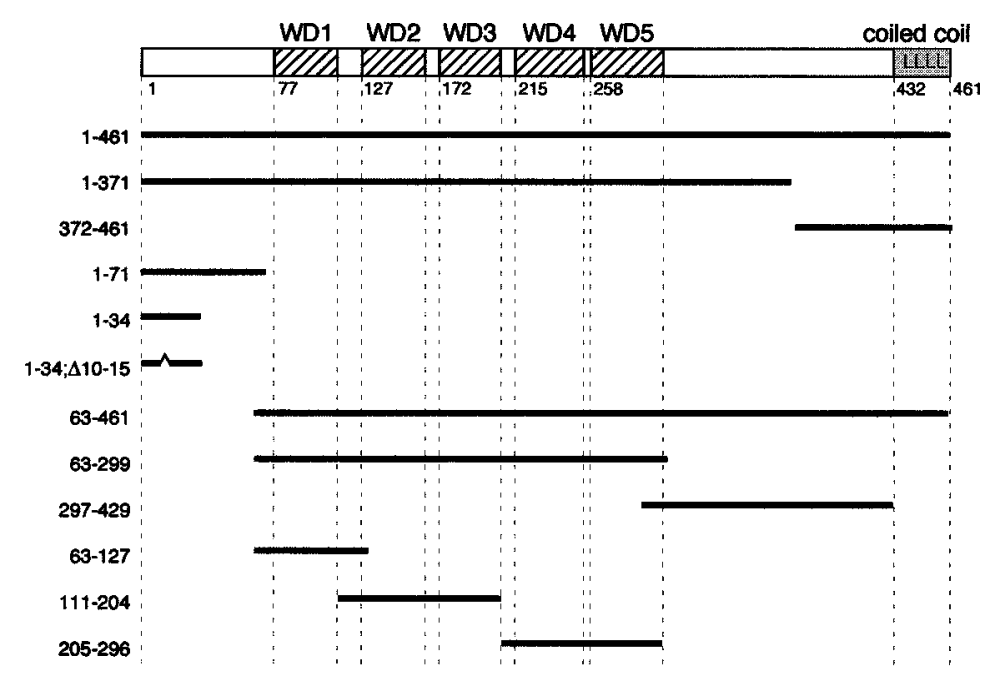

Fig. 1. Structures of p57 and its Deletions

The upper panel indicates the schematic representation of $\mathrm{p} 57$. Hatched boxes indicate WD repeats and a shaded box indicates a coiled-coil motif. 
were induced by overnight culture of the bacteria with $0.5 \mathrm{~mm}$ isopropylthio- $\beta$-D-galactoside. After the culture, the bacteria were pelleted, suspended in a sonication buffer (50 mM Tris-HCl, pH 8.0, $150 \mathrm{~mm} \mathrm{NaCl}, 1 \mathrm{~mm}$ EDTA), and incubated with $1.0 \mathrm{mg} / \mathrm{ml}$ of lysozyme for $20 \mathrm{~min}$ on ice. The suspensions were sonicated and centrifuged at $10000 \times \boldsymbol{g}$ for $30 \mathrm{~min}$. Then, the supernatants were incubated with $0.25 \mathrm{ml}$ of glutathione-Sepharose beads (Pharmacia) overnight at $4{ }^{\circ} \mathrm{C}$. The beads were washed three times with a sonication buffer. The bound GST fusion proteins were eluted with $50 \mathrm{~mm}$ reduced glutathione in $50 \mathrm{~mm}$ Tris- $\mathrm{HCl}$ buffer, $\mathrm{pH}$ 8.0.

Actin Co-sedimentation Assay An actin co-sedimentation assay was performed to investigate the ability of fusion proteins to bind actin. Each fusion protein, with or without G-actin (30 $\mu \mathrm{g}$, Sigma, St. Louis, MO, U.S.A.) was incubated in F-actin buffer (20 mM Tris-HCl, pH 8.0, $160 \mathrm{~mm} \mathrm{KCl,} \mathrm{and}$ $0.2 \mathrm{~mm} \mathrm{ATP}$ ) for $1.5 \mathrm{~h}$ at $25^{\circ} \mathrm{C}$. After the incubation, the reaction mixtures were ultracentrifuged at $198000 \times \boldsymbol{g}$ for $1.5 \mathrm{~h}$ at $4{ }^{\circ} \mathrm{C}$. The reaction mixture before ultracentrifugation is referred to as the total reaction mixture in the text. The supernatants, pellets, and total reaction mixtures were resolved by sodium dodecyl sulfate (SDS)-polyacrylamide gel electrophoresis (PAGE) (10\% gel), and gels were stained with Coomassie brilliant blue (CBB). To confirm the presence of fusion proteins, immunoblot analysis using anti-p57 polyclonal antibody ${ }^{4)}$ or anti-p57 monoclonal antibody ${ }^{9)}$ was also performed.

Expression Plasmids of p57 and Its Deletions To construct plasmids for the expression of p57 in mammalian cells, the DNA fragment encoding full-length p57 was amplified by PCR. The primers used were (restriction enzyme sites are underlined): 5'-GGG GAA TTC AGA ATG AGC CGG CAG GTG G-3' and 5'-GGG GAA TTC AAG CTT GGG GCT CTA CTT GGC CTG G-3'. The amplified DNA fragments were digested with EcoRI and cloned into pEF1/MycHis A (Invitrogen, Carlsbad, CA, U.S.A.). Then, TAG stop codon was replaced by GCG to generate a fusion protein with Myc-His tag using a QuikChange Site-directed mutagenesis kit (Stratagene). The primers used were: 5'-GGA GAC AGT CCA GGC CAA GGC GAG CCC CAA GCT TGA ATT CTG C-3' and 5'-GCA GAA TTC AAG CTT GGG GCT CGC CTT GGC CTG GAC TGT CTC C-3'. To generate the expression plasmid pcDNA3.1/p57-V5-His for fulllength p57, which expresses p57 fused with viral epitope V5 and $6 \mathrm{xHis}$ tag, the DNA fragments encoding p57 were subcloned into pcDNA3.1/V5-His A (Invitrogen).

For expression of $\mathrm{p} 57^{1-371}$ and $\mathrm{p} 57^{372-461}$, corresponding DNA fragments were subcloned into pcDNA3.1/V5His A (pcDNA3.1/p57 ${ }^{1-371}$ and pcDNA3.1/p57 $7^{372-461}$ ). Expression plasmids encoding $\mathrm{p} 57^{1-34}$ and $\mathrm{p} 57^{1-71}$ were generated from pcDNA3.1/p57-V5-His by introducing stop codons (pcDNA3.1/p57 $7^{1-34}$ and pcDNA3.1/p5 $57^{1-71}$, respectively).

The p5 $7^{111-204}$ was expressed as a fusion protein with an $\mathrm{N}$-terminal Xpress ${ }^{\mathrm{TM}}$ tag (Invitrogen) in order to facilitate detection of the expressed protein. The cDNA fragment of p5 $7^{111-204}$ was prepared by digestion of pGEX-p57 $7^{111-204}$ with BamHI and EcoRI, and subcloned into pcDNA3.1/His A (Invitrogen).

Cell Culture and Transfection COS-1 (monkey kidney cell line) cells (American Type Culture Collection) were grown in Iscove's Modified Dulbecco's Medium (IMDM, GIBCO) supplemented with $10 \%$ heat inactivated FCS (Sanko Junyaku Co., Ltd., Tokyo), penicillin (50 U/ml), and streptomycin $(50 \mu \mathrm{g} / \mathrm{ml})$ under the standard cell culture condition $\left(37^{\circ} \mathrm{C}\right.$, humidified $5 \% \mathrm{CO}_{2}$ in air).

Plasmids encoding p57 and its deletions were introduced into COS-1 cells by electroporation. Briefly, COS-1 cells $\left(4 \times 10^{6}\right.$ cells $\left./ 300 \mu \mathrm{l}\right)$ in phosphate buffered saline (PBS) were mixed with $10 \mu \mathrm{g}$ plasmid DNA and placed in a $0.4 \mathrm{~cm}$ electrode gap electroporation cuvette (Bio-Rad, Hercules, CA, U.S.A.). Cells were subjected to a single pulse of $750 \mathrm{~V} / \mathrm{cm}$ at a capacitance setting of 975 microfarads, transferred to $10 \mathrm{ml}$ of IMDM containing 10\% FCS, and maintained for $48 \mathrm{~h}$ under standard cell culture conditions.

Immunofluorescence Microscopy The transfected cells were cultured on a Lab-Tek II Chamber Slide (Nalge Nunc, Rochester, NY, U.S.A.) for $10 \mathrm{~min}$, fixed with $4 \%$ neutral buffered formaldehyde (Kanto Chemicals Co., Ltd., Tokyo, Japan), and then permeabilized by treatment with $0.2 \%$ Triton X-100 in PBS for $10 \mathrm{~min}$ at room temperature. Permeabilized cells were rinsed with PBS and incubated with a monoclonal antibody to $\mathrm{p} 57, \mathrm{~N} 7,{ }^{20)}$ which recognizes the C-terminal region of $\mathrm{p} 57$, at $5 \mu \mathrm{g} / \mathrm{ml}$ in PBS containing 3\% BSA for $1 \mathrm{~h}$. For the detection of N-terminal fragments of p57, a rabbit polyclonal antibody, which recognizes N-terminal 20 amino acids, was used. For the detection of Xpress-tagged fusion protein, an anti-Xpress antibody (1:5000, Invitrogen) was used. After being washed with PBS, the cells were incubated with FITC-labeled goat anti-mouse IgG (KPL, Gaithersburg, MD, U.S.A.) or FITC-labeled goat anti-rabbit IgG (KPL) for $30 \mathrm{~min}$. F-actin was detected by using rhodamine-conjugated phalloidin (Sigma). Fluorescently labeled cells were washed three times with PBS and then mounted with 2.3\% 1,4-diazabicycle-2,2,2-octane (Sigma) containing glycerol on slide glass. Samples were analyzed by using a confocal laser microscope (Radiance 2100 laser scanning system, Bio-Rad, Hercules, CA, U.S.A.).

\section{RESULTS}

Identification of Region(s) in p57 Responsible for Actin Binding To identify the region(s) of p57 responsible for actin binding, we examined the abilities of GST-fusion proteins with full-length and several truncated forms of p57 to co-sediment with F-actin in vitro. GST-p57 (a fusion protein of full-length p57 with GST) and GST-p57 ${ }^{1-371}$ (a fusion protein containing five WD repeats but lacking a C-terminal coiled-coil motif) (Fig. 1) co-precipitated with F-actin (Figs. 2A, B). Although some impurities were present in the GSTp57 preparation, the identity of GST-p57 was confirmed by immunoblotting using an anti-p57 antibody, and the impurities remained in the supernatant after ultracentrifugation. In contrast, GST-p57 $7^{372-461}$ (a fusion protein containing a C-terminal fragment with a coiled-coil motif) (Fig. 1) did not cosediment with F-actin (Fig. 2C). These results suggested that the region responsible for $\mathrm{p} 57$ binding to actin was located in the N-terminal part of p57 that includes WD repeats, but does not include the coiled-coil motif at the C-terminus. We then prepared two additional truncated proteins containing the N-terminus of p57 but lacking the WD repeats, GSTp5 $7^{1-71}$ and GST-p5 $7^{1-34}$ (Fig. 1). These deletion mutants 
A

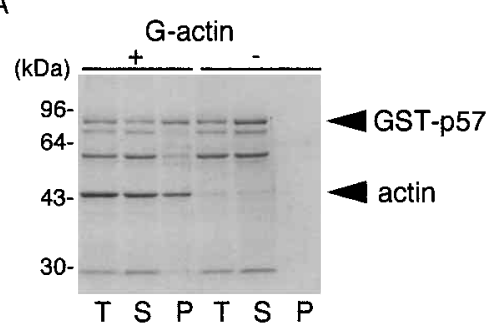

C

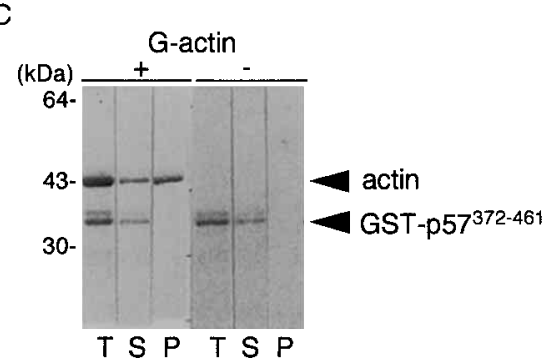

B

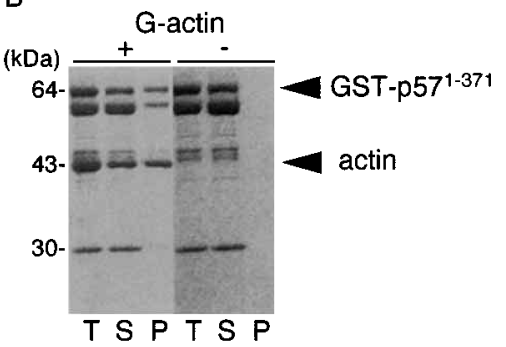

Fig. 2. Co-sedimentation Assay of GST-p57 (A), GST-p $57^{1-371}$ (B), and GST-p57 $7^{372-461}$ (C) Fusion Proteins with F-Actin

The supernanant $(\mathrm{S})$ and the precipitate $(\mathrm{P})$ after ultracentrifugation, as well as the total reaction mixture $(\mathrm{T})$, were separated on SDS-polyacrylamide gel electrophoresis. Two sets of samples in the presence $(+)$ or absence $(-)$ of G-actin were assayed.

\section{A p57 1.34 MSRQVVRSSK FRHVFGQPAK ADQCYEDVRV SQTT p571-34;A10-15 MSRQVVRSS----------FGQPAK ADQCYEDVRV SQTT}

B

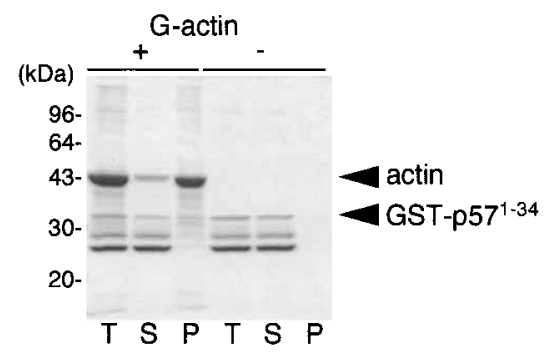

C

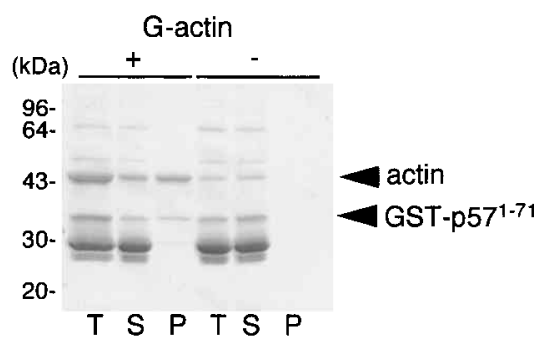

D

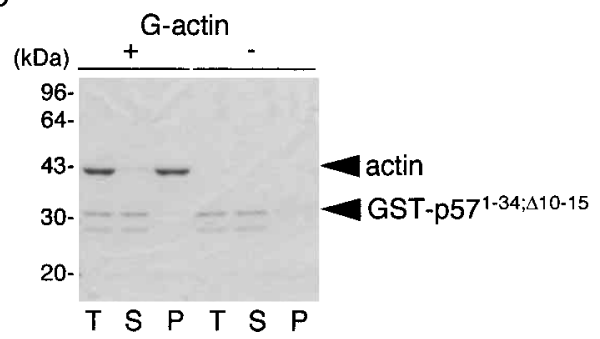

Fig. 3. Amino Acid Sequences of GST-p $57^{1-34}$ and GST-p $57^{1-34 ; 410-15}$ (A) and the Co-sedimentation Assay of GST-p $57^{1-34}$ (B), GST-p 57 ${ }^{1-71}$ (C), and GST-p5 $7^{1-34 ; 410-15}$ (D) Fusion Proteins with F-Actin

After F-actin was sedimented by ultracentrifugation, each fraction was subjected to SDS-polyacrylamide gel electrophoresis; T, total reaction mixture; S, ultracentrifuged supernatant; $\mathrm{P}$, ultracentrifuged precipitate. Each fusion protein, with $(+)$ or without $(-)$ G-actin, was incubated in F-actin buffer for co-sedimentation assay.

co-precipitated with F-actin (Figs. 3B, C), suggesting that the N-terminal 34 amino acids of p57 possessed the capacity to bind F-actin. Moreover, the deletion of six amino acid residues, ${ }^{10} \mathrm{KFRHVF}^{15}$, from $\mathrm{p} 57^{1-34}$ (GST-p57 ${ }^{1-34 ; 410-15}$ ) abolished the actin-binding ability (Fig. 3D), implicating a sequence rich in basic and hydrophobic amino acids as essential for actin binding of $\mathrm{p} 57^{1-34}$.

Further Analysis of Region(s) in p57 Responsible for
Actin Binding To evaluate the role of the central region of p57 molecules containing WD repeats in actin binding, we analyzed the F-actin binding capacity of six truncated fusion proteins of $\mathrm{p} 57$ that lack the putative N-terminal actin-binding sequence, namely GST-p $57^{63-461}$, GST-p $57^{63-299}$, GSTp5 $7^{297-429}$, GST-p57 $7^{63-127}$, GST-p5 $7^{111-204}$ and GST-p57 $7^{205-}$ ${ }_{296}$ (Fig. 1). GST-p57 ${ }^{63-461}$, which lacks the N-terminal 62 amino acids, co-precipitated with F-actin (Fig. 4A), suggest- 
A

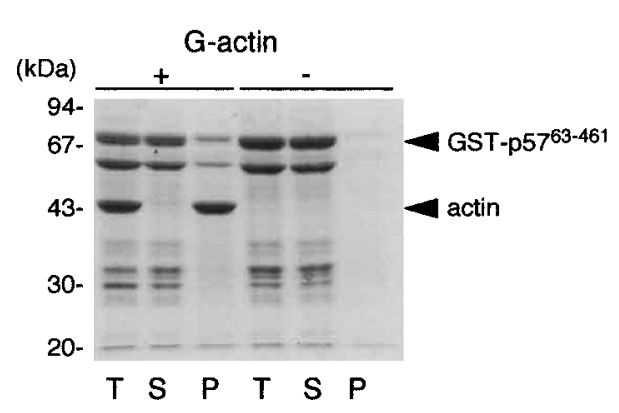

C

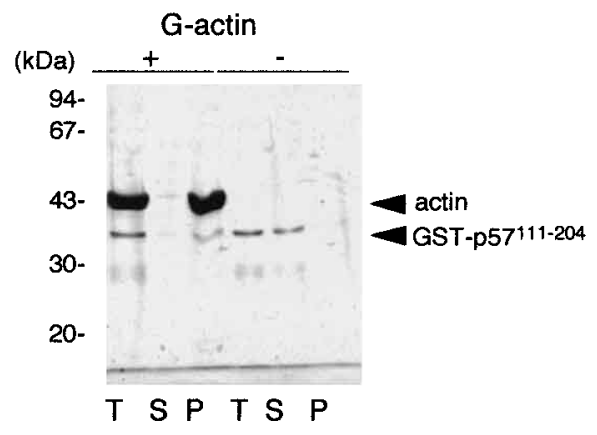

$E$

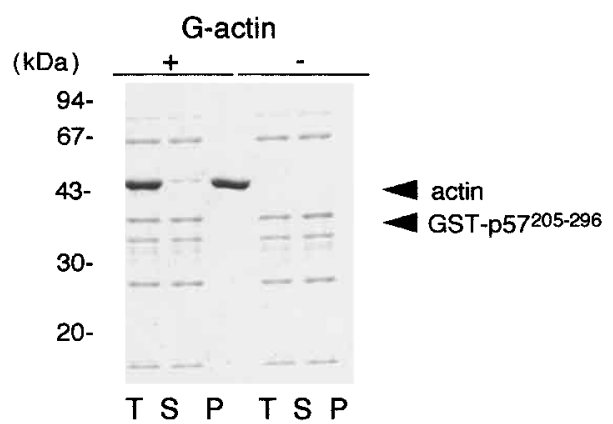

Fig. 4. Co-sedimentation Assay of GST-p57 $63-461$ (A), GST-p57 $7^{63-299}$ p5 $7^{297-429}$ (F) Fusion Proteins with F-Actin

After F-actin was sedimented by ultracentrifutgation, each fraction was subjected to SDS-polyacrylamide gel electrophoresis; T, total reaction mixture; S, ultracentrifuged supernatant, $\mathrm{P}$, ultracentrifuged precipitate. Each fusion protein, with $(+)$ or without $(-) \mathrm{G}$-actin was incubated in F-actin buffer for co-sedimentation assay.

ing the presence of a second region responsible for actinbinding of $\mathrm{p} 57$ in addition to the $\mathrm{N}$-terminal sequence $\left(\mathrm{p} 57^{1-34}\right)$. Furthermore, GST-p57 $7^{63-299}$ and GST-p57 $7^{111-204}$ also co-precipitated with F-actin (Figs. 4B, C), whereas GST-p57 $7^{63-127}$, GST-p57 $7^{205-296}$, and GST-p57 $7^{297-429}$ did not (Figs. 4D-F). Based on these results on the differential representation of the WD repeats among these mutant forms of p57 (Fig. 1), it is strongly suggested that p57 contains at least two regions that mediate actin binding; i.e., the $\mathrm{N}$-terminal region $\left(\mathrm{p} 57^{1-34}\right)$ and the central region containing the second and third WD repeats $\left(\mathrm{p} 57^{111-204}\right)$.

Binding of p57 Deletions to Actin in COS-1 Cells To extend our analysis of the putative actin-binding regions of p57 to intact cells, we expressed in COS-1 cells full-length
B

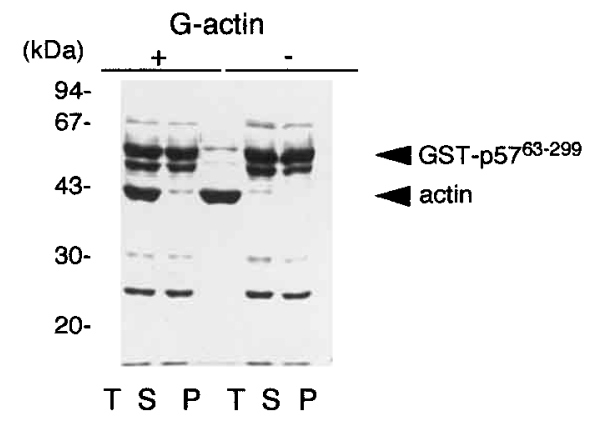

D

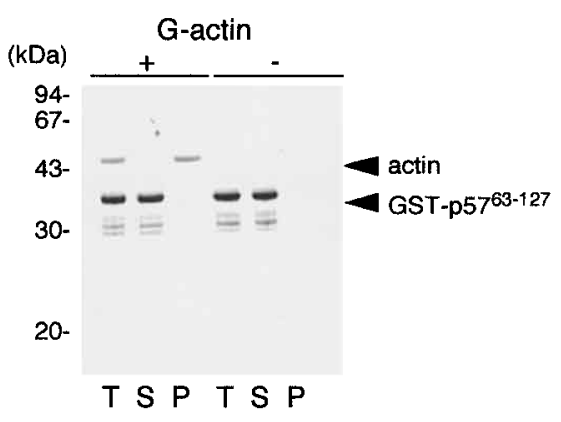

$\mathrm{F}$

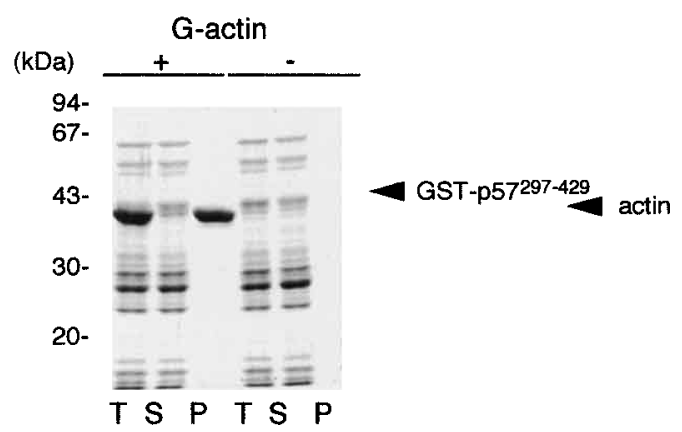

and deletion mutants of $\mathrm{p} 57$ ( $\mathrm{p} 57^{1-371}, \mathrm{p} 57^{372-461}, \mathrm{p} 57^{1-71}$, $\mathrm{p} 57^{1-34}$ and $\mathrm{p} 57^{111-204}$ ). Expressed forms of p57 constructs were visualized in transiently transfected cells using immunofluorescence microscopy (Fig. 5). Full-length p57 and its deletions that exhibited actin-binding activity in vitro, including $\mathrm{p} 57^{1-371}, \mathrm{p} 57^{1-71}, \mathrm{p} 57^{1-34}$ and $\mathrm{p} 57^{111-204}$, were localized in cortical F-actin rich regions. In contrast, p57 $7^{372-461}$, which did not exhibit actin-binding activity in vitro, was diffusely distributed in cytosol and did not show cortical localization. These results are in good agreement with those of the co-sedimentation assay in vitro. 


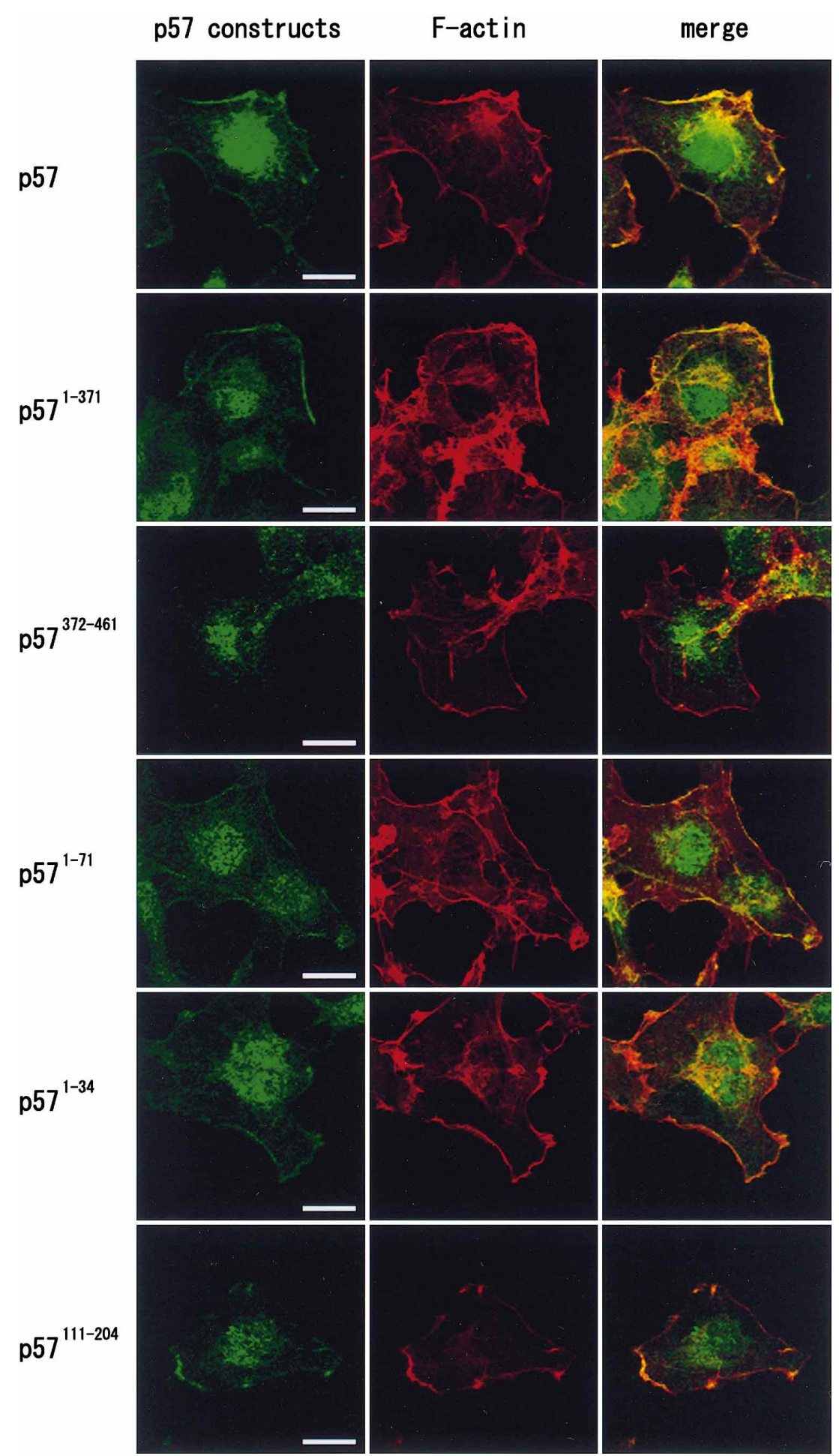

Fig. 5. Intracellular Localization of p57 and Its Deletions Expressed in COS-1 Cells

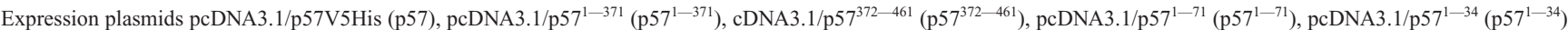
and pcDNA3.1/p5 $7^{111-204}\left(\mathrm{p} 57^{111-204}\right)$ were transiently expressed in COS-1 cells. Deletion mutants of p57 and F-actin were stained by anti-p57 antibodies or anti-Xpress antibody, followed by secondary antibody conjugated with FITC and by rhodamine-labelled phalloidin, respectively, and they were observed by fluorescence microscopy. Experiments were performed more than three times, and representative data are shown. Scale bars, $20 \mu \mathrm{m}$.

\section{DISCUSSION}

Our previous study demonstrated that the transient periphagosomal association of $\mathrm{p} 57$ plays an essential role in the maturation of phagolysosome in phagocytes. ${ }^{24)}$ Considering that reorganization of the actin cytoskeleton is required for phagocytosis, the interaction between $\mathrm{p} 57$ and F-actin is one of the key steps during phagocytic processes. In this study, we have demonstrated that a p57 molecule possesses two regions responsible for its binding to F-actin. One is located in a short stretch of N-terminal 34 amino acids, and the other is located in the central region of the molecule containing WD repeats. Our results are partly consistent with a recent report 


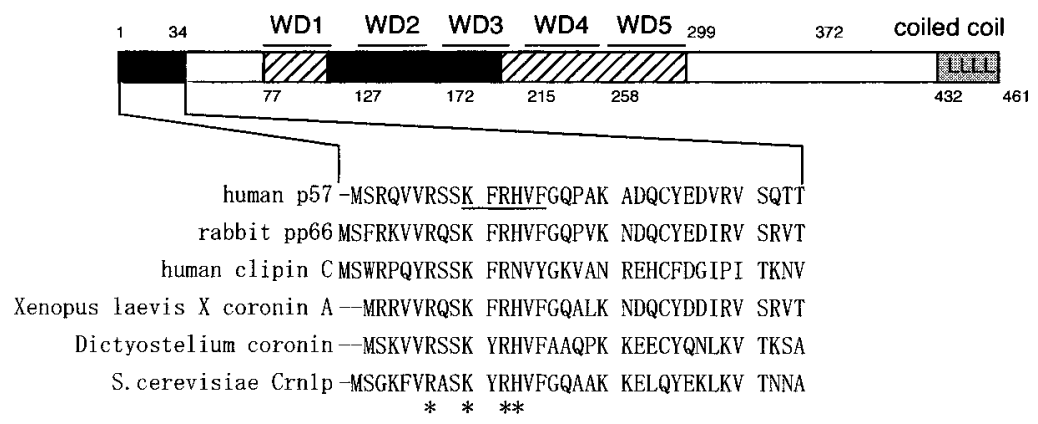

Fig. 6. Alignment of the Putative Actin-binding Region at the N-terminus of p57 and the Corresponding Regions of Other Coronin Family Actin-Binding Proteins

Asterisks indicate basic amino acid residues conserved in the coronin family of actin-binding proteins.

by Goode $e t$ al. ${ }^{8)}$ They demonstrated that the actin-binding region of a yeast homologue of coronin, Crn1p, was located in the N-terminal part containing WD repeats, but not in its C-terminal region with a coiled-coil motif. By contrast, Mishima and Nishida ${ }^{13)}$ reported that the deletion of N-terminal 63 amino acids or of C-terminal 65 amino acids from Xcoronin, a Xenopus homologue of coronin, reduces, but does not eliminate, its actin-binding activity. The reduction of actin-binding activity in Xcoronin lacking its C-terminal coiled-coil motif is not consistent with the results from p57 and Crn1p. This apparent conflict may be reflected by the differences among coronin species, and coronin homologues do not necessarily share common characteristics in the actinbinding activity.

A co-sedimentation assay and fluorescent microscopic observation strongly suggest that the N-terminal fragment of p57 with 34 amino acids $\left(\mathrm{p} 57^{1-34}\right.$ ) was bound to F-actin (Figs. 3, 5). Deletion of the sequence ${ }^{10} \mathrm{KFRHVF}^{15}$ abolished the actin-binding activity of $\mathrm{p} 57^{1-34}$, suggesting that the stretch of these six amino acids is essential for actin binding of the N-terminal fragment. Several known actin-binding proteins possess regions rich in basic amino acid residues that contribute to actin binding through their interaction with acidic amino acid clusters contained in actin. ${ }^{25-28)}$ This cluster of basic amino acid residues may be responsible not only for actin binding by $\mathrm{p} 57$, but may also represent a motif common to related proteins, as the KXRHXX (where X represents a hydrophobic amino acid) is conserved in many other coronin-family actin-binding proteins (Fig. 6).

In addition to $\mathrm{p} 57^{1-34}$, we identified a second region of p57 that exhibited actin-binding activity with Ile-111 to Glu204 (Fig. 4). p5 $7^{111-204}$ includes two out of the five WD repeats present in $\mathrm{p} 57$. The WD motifs were found in a variety of proteins and are known to participate in various critical cell functions, including signal transduction, gene regulation, vesicular trafficking, regulation of the cytoskeleton and the cell cycle. ${ }^{29)}$ Regions in proteins with WD repeats are thought to organize into a $\beta$-propeller structure and to promote protein-protein interactions. ${ }^{30,31)}$ However, the WD repeats in p57 do not seem to mediate its actin-binding, but more likely serve another function, perhaps providing a scaffold for the responsible regions of $p 57$ to bind actin and/or link actin to another protein. In the case of $\mathrm{p} 57^{111-204}$ there are WD repeats sufficient for only one blade of a $\beta$-propeller, thus it is inadequate to fold properly into a functional $\beta$-propeller structure. ${ }^{30)}$ Therefore, the actin binding of $\mathrm{p} 57^{111-204}$ more likely depends on a structure other than the WD repeats. The determination of the second sequence responsible for the binding of p5711-204 to F-actin awaits further investigation.

The presence of two actin-binding regions in a p57 molecule would increase its affinity for F-actin, as multiple actinbinding regions in several actin-binding proteins are believed to augment their interaction with actin. This hypothesis is supported by the result that the interaction of intact $\mathrm{p} 57$ with F-actin seems to be stronger than those of deletions containing only one of two actin-binding regions (Figs. 2, 4).

Acknowledgements This work was supported by the Ministry of Education, Culture, Sports, Science and Technology of Japan, the Ministry of Health, Labour and Welfare of Japan, and the U.S. Public Health Service (RO1AI34879).

\section{REFERENCES}

1) Nauseef W. M., Clark R. A., "Principles and Practice of Infectious Diseases," 5th ed., Chap. 8, eds. by Mandell G. L., Bennett J. E., Dolin R., Churchill Livingstone, Philadelphia, 2000, pp. 89-111.

2) May R. C., Machesky L. M., J. Cell Sci., 114, 1061-1077 (2001).

3) Noegel A. A., Schleicher M., J. Cell Sci., 113, 759-766 (2000).

4) Suzuki K., Nishihata J., Arai Y., Honma N., Yamamoto K., Irimura T., Toyoshima S., FEBS Lett., 364, 283-288 (1995).

5) de Hostos E. L., Bradtke B., Lottspeich F., Guggenheim R., Gerisch G., EMBO J., 10, 4097-4104 (1991).

6) de Hostos E. L, Rehfuess C., Bradtke B., Waddell D., R., Albrecht R., Murphy J., Gerisch G., J. Cell Biol., 120, 163-173 (1993).

7) Maniak M., Rauchenberger R., Albrecht R., Murphy J., Gerisch G., Cell, 83, 915-924 (1995).

8) Goode B. L., Wong J. J., Butty A. C., Peter M., McCormack A. L., Yates J. R., Drubin D. G., Barnes G., J. Cell Biol., 144, 83—98 (1999).

9) Tardieux I., Liu X., Poupel O., Parzy D., Dehoux P., Langsley G., FEBS Lett., 441, 251-256 (1998).

10) Bricheux G., Coffe G., Bayle D., Brugerolle G., Eur. J. Cell Biol., 79, $413-422(2000)$.

11) Rappleye C. A., Paredez A. R., Smith C. W., McDonald K. L., Aroian R. V., Genes Dev., 13, 2838-2851 (1999).

12) Terasaki A. G., Ohnuma M., Mabuchi I., J. Biochem. (Tokyo), 122, 226-236 (1997).

13) Mishima M., Nishida E., J. Cell Sci., 112, 2833-2842 (1999).

14) Iizaka M., Han H. J., Akashi H., Furukawa Y., Nakajima Y., Sugano S., Ogawa M., Nakamura Y., Cytogenet. Cell Genet., 88, 221-224 (2000)

15) Nakamura T., Takeuchi K., Muraoka S., Takezoe H., Takahashi N., Mori N., J. Biol. Chem., 274, 13322-13327 (1999).

16) Parente J. A., Jr., Chen X., Zhou C., Petropoulos A. C., Chew C. S., J. Biol. Chem., 274, 3017-3025 (1999).

17) Okumura M., Kung C., Wong S., Rodgers M., Thomas M. L., DNA Cell Biol., 17, 779-787 (1998). 
18) de Hostos E. L., Trends Cell Biol., 9, 345-350 (1999).

19) Grogan A., Reeves E., Keep N., Wientjes F., Totty N. F., Burlingame A. L., Hsuan J. J., Segal A. W., J. Cell Sci., 110, 3071-3081 (1997).

20) Allen L. A., DeLeo F. R., Gallois A., Toyoshima S., Suzuki K., Nauseef W. M., Blood, 93, 3521-3530 (1999).

21) Ferrari G., Langen H., Naito M., Pieters J., Cell, 97, 435-447 (1999).

22) Cohen D. R., Curran T., Mol. Cell. Biol., 8, 2063-2069 (1988).

23) Vinson C. R., Hai T. W., Boyd S., Genes. Dev., 7, 1047 -1058 (1993).

24) Itoh S., Suzuki K., Nishihata J., Iwasa M., Oku T., Nakajin S., Nauseef W. N., Toyoshima S., Biol. Pharm. Bull., 25, 837-844 (2002).

25) Lappalainen P., Fedorov E. V, Fedorov A. A, Almo S. C., Drubin D. G., EMBO J., 16, 5520—5530 (1997).

26) Yao L., Janmey P., Frigeri L. G., Han W., Fujita J., Kawakami Y.,
Apgar J. R., Kawakami T., J. Biol. Chem., 274, 19752-19761 (1999).

27) de Arruda M. V., Bazari H., Wallek M., Matsudaira P., J. Biol. Chem., 267, 13079-13085 (1992).

28) Jongstra-Bilen J., Janmey P. A., Hartwig J. H, Galea S., Jongstra J., J. Cell Biol., 118, 1443-14453 (1992).

29) Neer E. J., Schmidt C. J., Nambudripad R., Smith T. F., Nature (London), 371, 297-300 (1994).

30) Sondek J., Bohm A., Lambright D. G., Hamm H. E., Sigler P. B., Nature (London), 379, 369-374 (1996).

31) Garcia-Higuera I., Fenoglio J., Li Y., Lewis C., Panchenko M. P., Reiner O., Smith T. F., Neer E. J., Biochemistry, 35, 13985-13994 (1996). 